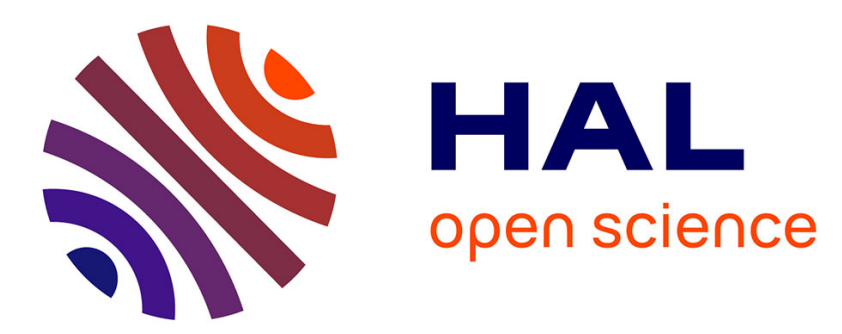

\title{
A multi-scale analysis of materials reinforced by inclusions randomly oriented in the ply plane
}

Emmanuel Lacoste, Sylvain Fréour, Frédéric Jacquemin

\section{To cite this version:}

Emmanuel Lacoste, Sylvain Fréour, Frédéric Jacquemin. A multi-scale analysis of materials reinforced by inclusions randomly oriented in the ply plane. Advanced Materials Research, 2011, 61, pp.55-64. 10.4028/www.scientific.net/AMM.61.55 . hal-01004854

\section{HAL Id: hal-01004854 https://hal.science/hal-01004854}

Submitted on 26 May 2018

HAL is a multi-disciplinary open access archive for the deposit and dissemination of scientific research documents, whether they are published or not. The documents may come from teaching and research institutions in France or abroad, or from public or private research centers.
L'archive ouverte pluridisciplinaire HAL, est destinée au dépôt et à la diffusion de documents scientifiques de niveau recherche, publiés ou non, émanant des établissements d'enseignement et de recherche français ou étrangers, des laboratoires publics ou privés. 


\title{
A multi-scale analysis of materials reinforced by inclusions randomly oriented in the ply plane
}

\author{
Emmanuel Lacoste $^{a}$, Sylvain Fréour ${ }^{b}$, Frédéric Jacquemin ${ }^{c}$ \\ Institut de Recherche en Génie Civil et Mécanique (UMR CNRS 6183), Université de Nantes - \\ Centrale Nantes, 37 Boulevard de I'Université, BP 406, 44602 Saint-Nazaire cedex, France \\ aemmanuel.lacoste@univ-nantes.fr, ${ }^{b}$ sylvain.freour@univ-nantes.fr, \\ cfrederic.jacquemin@univ-nantes.fr
}

Key words: composite materials, multiscale analysis, Kröner - Eshelby self-consistent model, multiple inclusions, random orientation, thermoelastic behaviour.

Abstract. The present work aims to investigate the validity of Eshelby-Kröner self-consistent model [1] for thermoelastic behaviour, in the case of a material reinforced by inclusions randomly oriented in the ply plane. The model provides predictive information on the properties and multi-scale mechanical states experienced by the material, accounting for its constituents properties, but also their morphology. However, it cannot reliably account for multiple inclusion morphologies (shape and orientation) in the material [2-4]. A study of the two applicable formulations and their limits leads to suggest a mixed formulation as an acceptable compromise between those alternatives. The results of this original approach are also described in the case of a thermo-mechanical load.

\section{Introduction}

The recent development of composite materials during the last decades opened new prospects to mechanical part engineering, particularly for aeronautical applications, because of their high strength-to-weight ratio as well as corrosion and fatigue resistance. However, the inherent heterogeneity of these materials induces internal stresses during the curing process, and complex mechanical behaviour under service loads. The so-called "scale transition models" answer the necessity to predict the distribution of stresses between the constituents of the composite plies. Among these, the self-consistent model for the polycrystals [1] suggests a realistic and interesting approach; it enables one to calculate the homogenized properties of the material, but also the mechanical states experienced by the constituents, accounting for their morphology and properties.

The present application is focused on an in-plane isotropic composite material [5] made of unidirectional reinforcing strips with rectangular shape $(60 \times 8 \times 0.15 \mathrm{~mm})$ and randomly disposed in the ply plane (see Fig. 1 below). The strips themselves are composed of an unidirectional (UD) carbon-organic ply. An N5208 epoxy matrix and T300 carbon fibers were considered for this study.

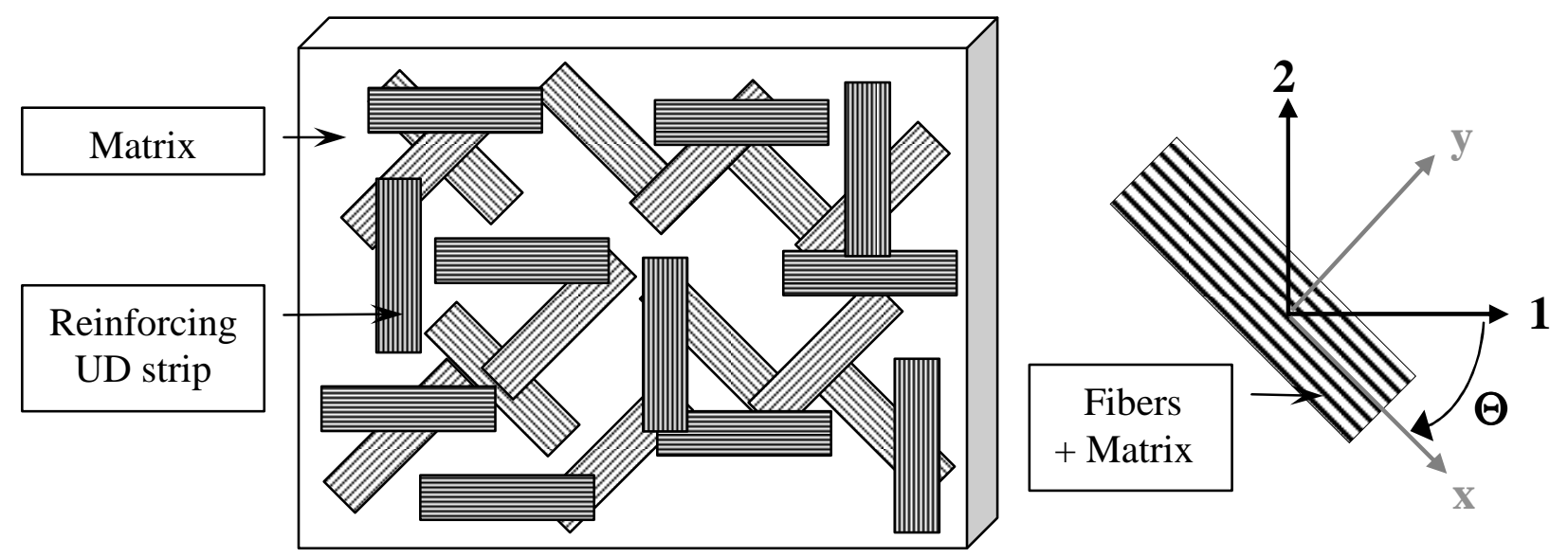

Figure 1: Schematic representation of the microstructure of the composite 
The self-consistent model is used twice in our approach, in order to perform a two-steps scale transition procedure (Fig. 2). First, the effective properties of the reinforcing strip are estimated from those of the intra-reinforcements matrix and the carbon fibers. These effective properties are then used for the second homogenization step, in order to find those of the whole composite.

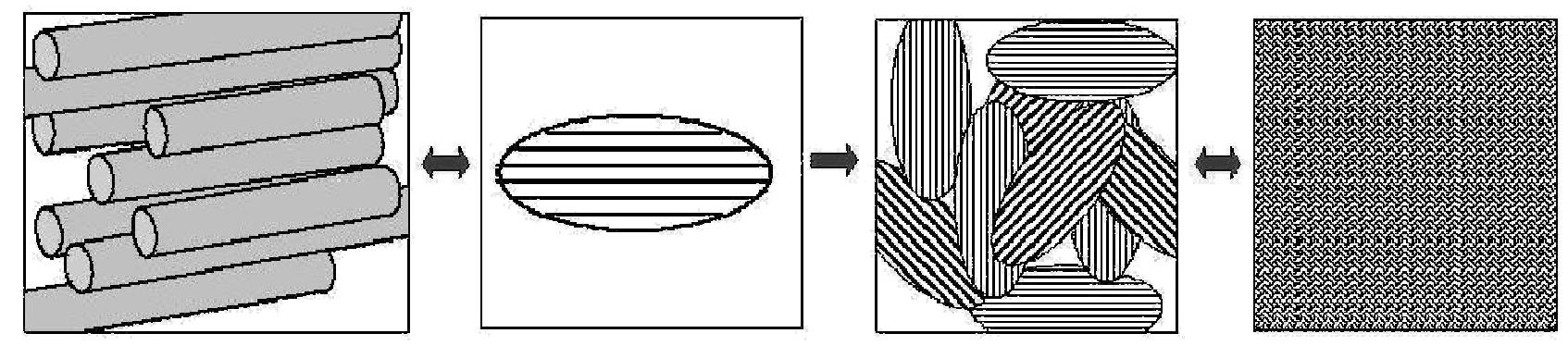

Figure 2: Schematic representation of the two steps scale transition procedure

\section{The self-consistent model}

Hill's formalism and Eshelby's inclusion. Scale transition models are based on a representation of the material at several scales: on the one hand, the "local" scale denoted by the superscript i, where one observes the behaviour of each constituent, considered as an ellipsoidal and homogeneous inclusion (also called Base Volume or BV). On the other hand, the macroscopic scale, denoted by the superscript I, defines the behaviour of the Effective Medium (EM). A linear thermoelastic law (Eq. 1) expresses those behaviors. In this relation, the stiffness is represented by the $4^{\text {th }}$ order tensor $\mathbf{L}$, and the Coefficients of Thermal Expansion (CTE) by the $2^{\text {nd }}$-order tensor $\boldsymbol{\alpha}$. The temperature increment is denoted by $\Delta \mathrm{T}$, whereas $\boldsymbol{\sigma}$ and $\boldsymbol{\varepsilon}$ stand respectively for the stress and strain.

$$
\sigma^{\mathbf{k}}=\mathbf{L}^{\mathbf{k}}:\left(\varepsilon^{\mathbf{k}}-\alpha^{\mathrm{k}} \Delta \mathrm{T}\right), \mathrm{k}=\mathrm{i}, \mathrm{I}
$$

Hill [6] demonstrated, in a very general way, the equivalence between volume integrals and set (i.e. volume fraction weighted) averages, denoted here by angle brackets <...>. The semi-statistical ("mean-field") approach studied here uses Hill's volume average relations over the mechanical states, written as in the equations below (Eq. 2, Eq. 3):

$$
\begin{aligned}
& \varepsilon^{I}=\left\langle\varepsilon^{i}\right\rangle, \\
& \sigma^{I}=\left\langle\sigma^{i}\right\rangle .
\end{aligned}
$$

In a fundamental work, Eshelby [7] determined the behavior of an inclusion embedded in a homogeneous medium, loaded at the infinite. He demonstrated that, if the inclusion had an ellipsoidal shape, the local stresses and strains were homogeneous inside the BV. Using this work, Hill [8] proposed the following relation (Eq. 4) between local and overall states, where $\mathbf{L}^{*}$ is widely known as Hill's constraint tensor.

$$
\begin{aligned}
& \sigma^{\mathrm{i}}-\boldsymbol{\sigma}^{\mathrm{I}}=-\mathbf{L}^{*}:\left(\boldsymbol{\varepsilon}^{\mathrm{i}}-\boldsymbol{\varepsilon}^{\mathrm{I}}\right), \text { with } \\
& \mathbf{L}^{*}=\mathbf{L}^{\mathrm{I}}:\left(\mathbf{S}^{\mathbf{I}^{-1}}-\mathbf{I}^{(\mathbf{4})}\right)=\left(\mathbf{E}^{\mathbf{I}^{-1}}-\mathbf{L}^{\mathrm{I}}\right) .
\end{aligned}
$$

This tensor can be obtained from the Eshelby tensor $\mathbf{S}^{\mathbf{I}}$ or the Morris tensor $\mathbf{E}^{\mathbf{I}}$ [1, 9] (also referred to "influence tensor" $\mathbf{P}^{\mathbf{I}}$ ), thanks to Eq. 5 (with $\mathbf{I}^{(\mathbf{4})}$ the $4^{\text {th }}$-order Identity tensor). In the general case, the computation of the Morris tensor is a key-point of the model, as it implies several tensorial inversions and a numerical integration. Yet, analytical expressions can be found in the literature for 
a few specific configurations [10]. Morris' tensor depends on the stiffness of the embedding medium, and most importantly, on the shape ratios and orientation of the elementary inclusions. This dependency allows one to take into account the morphology of the constituents, i.e., to represent the microstructure of the considered material. Thus, the material anisotropies yielded by the microstructure (in glass fiber unidirectional plies, for instance) can be taken into account straightforwardly, which is a great advantage over to the more classical "rules of mixtures".

Several models are derived from these equations. The first and simplest one is the "dilute approximation", where one inclusion interacts with an embedding medium. The Mori-Tanaka scheme is an extent of this approximation to non-dilute inclusions, where one of the constituents (often called the "matrix phase") is considered as the embedding medium; the model is mainly adapted for materials whose matrix phase volume fraction dominates all the others. On the contrary, within the self-consistent model, the embedding medium is given the properties of the EM, which makes the expressions of the effective stiffness implicit. Yet, this approach is more adapted than Mori-Tanaka's for materials where all the phases have comparable volume fractions, as the EM yields a better emulation of the inter-particle interactions than the resin [11]. At high reinforcement ratios, the self-consistent model gives more reliable information than the Mori-Tanaka model upon the effective properties, particularly over the shear moduli. For this reason, the self-consistent model should be preferred to the Mori-Tanaka model to perform the second homogenization step (mesomacro), where one cannot define a dominating medium.

Eshelby's equations were first used by Kröner for estimating the elastic moduli and the plastic behaviour of polycrystals [12, 13], from the properties of its constitutive crystallites. This elastoplastic model inspired similar studies on the thermoelastic behaviour of heterogeneous materials [14], then on the time-dependent creep and relaxation of polycrystals [15]. They were also found interesting for composite materials with organic [10,13] or metallic matrix [16], owing to the strong heterogeneity of their constituents.

Formulation with stresses and strains. If the inclusions constituting the material do not present a single morphology in the macroscopic coordinate system RI, the tensor $\mathbf{L}^{*}$ is not purely macroscopic anymore but related to each inclusion (and in consequence, denoted $\mathbf{L}^{* \mathbf{i}}$ ). Then, using (Eq. 1) and (Eq. 4), one can express the local stresses and strains as:

$$
\begin{aligned}
& { }_{R i} \varepsilon^{i}=\left(L^{i}+L^{* i}\right)^{-1}:\left[\left(L^{I}+L^{* i}\right): \varepsilon^{I}+\left(L^{i}: \alpha^{i}-L^{I}: \alpha^{I}\right) \Delta T\right]=A^{i}: \varepsilon^{I}+a^{i} \cdot \Delta T \text {, and } \\
& { }_{R i} \sigma^{i}=L^{i}:\left(L^{i}+L^{* i}\right)^{-1}:\left[\left(L^{I}+L^{* i}\right): L^{I^{-1}}: \sigma^{I}+L^{* i}:\left(\alpha^{I}-\alpha^{i}\right) \Delta T\right]=B^{i}: \sigma^{I}+b^{i} \cdot \Delta T
\end{aligned}
$$

where $\mathbf{A}^{\mathbf{i}}$ is the elastic strain localization tensor, $\mathbf{B}^{\mathbf{i}}$ is the elastic stress concentration tensor, and $\mathbf{a}^{\mathbf{i}}$ and $\mathbf{b}^{\mathbf{i}}$ are the thermal strain and stress polarization tensors, respectively. Of course, Hill's averages principles imply and the following relation over the averages of $\mathbf{A}^{\mathbf{i}}, \mathbf{B}^{\mathbf{i}}, \mathbf{a}^{\mathbf{i}}$ and $\mathbf{b}^{\mathbf{i}}$ (Eq. 8, Eq. 9), where $\mathbf{0}^{(\mathbf{2})}$ represents the $2^{\text {nd }}$-order null tensor:

$$
\begin{aligned}
& \left\langle\mathbf{A}^{\mathbf{i}}\right\rangle=\mathbf{I}^{(4)} \text { and }\left\langle\mathbf{a}^{\mathbf{i}}\right\rangle=\mathbf{0}^{(2)} \\
& \left\langle\mathbf{B}^{\mathbf{i}}\right\rangle=\mathbf{I}^{(4)} \text { and }\left\langle\mathbf{b}^{\mathbf{i}}\right\rangle=\mathbf{0}^{(2)}
\end{aligned}
$$

Hill's averages principles are expressed over both strains and stresses. Nevertheless, in the classical self-consistent scheme, only one relation is needed in order to obtain the effective properties, which leads to two alternate expressions. Actually, using Hill's average principle over the stresses (formulation denoted $\mathrm{H} \sigma$ ), one obtains the following stiffness and CTE given in 
(Eq. 10) and (Eq. 11). On the contrary, if one uses Hill's average principle over the strains (formulation denoted $\mathrm{H} \varepsilon$ ), one would obtain the effective properties given in (Eq. 12) and (Eq. 13).

$$
\begin{aligned}
& { }^{H} \boldsymbol{\sigma}_{\mathbf{L}^{\mathbf{I}}}=\left\langle\mathbf{L}^{\mathbf{i}}: \mathbf{A}^{\mathbf{i}}\right\rangle \\
& { }^{\text {нб}} \boldsymbol{\alpha}^{\mathbf{I}}=\left\langle\mathbf{L}^{\mathrm{i}}:\left(\mathbf{L}^{\mathrm{i}}+\mathbf{L}^{* \mathrm{i}}\right)^{-1}: \mathbf{L}^{* \mathrm{i}}\right\rangle^{-1}:\left\langle\mathbf{L}^{\mathrm{i}}:\left(\mathbf{L}^{\mathrm{i}}+\mathbf{L}^{*^{\mathrm{i}}}\right)^{-1}: \mathbf{L}^{* \mathrm{i}}: \boldsymbol{\alpha}^{\mathrm{i}}\right\rangle \\
& { }^{\mathrm{H}} \boldsymbol{E}_{\mathbf{L}^{\mathrm{I}}}=\mathbf{L}^{\mathrm{I}}:\left\langle\mathbf{A}^{\mathrm{i}}\right\rangle^{-1}=\left\langle\mathbf{L}^{\mathrm{i}^{-1}}: \mathbf{B}^{\mathrm{i}}\right\rangle^{-1}, \\
& { }^{\text {не}} \alpha^{\mathrm{I}}=\mathbf{L}^{\mathrm{I}^{-1}}:\left\langle\left(\mathbf{L}^{\mathrm{i}}+\mathbf{L}^{*^{\mathrm{i}}}\right)^{-1}\right\rangle^{-1}:\left\langle\left(\mathbf{L}^{\mathrm{i}}+\mathbf{L}^{* \mathrm{i}}\right)^{-1}: \mathbf{L}^{\mathrm{i}}: \boldsymbol{\alpha}^{\mathrm{i}}\right\rangle .
\end{aligned}
$$

Self-consistency of the model. Several authors have shown that the two above formulations are equivalent, but with some restrictions over the materials microstructure: either the inclusions must have the same morphology (shape and alignment), or the material and inclusions must be isotropic. Analytical expressions of the thermo-elastic macroscopic properties of the EM have been determined for these specific cases in a series of paper from Benveniste, Dvorak and Chen [3, 4]. These configurations match many industrial applications, and in particular polycrystals and unidirectional composites plies.

Similar models have also been applied to new industrial materials that present a microstructure containing anisotropic inclusions of various morphologies or geometrical orientations [17]. However, for such microstructures, Benveniste, Dvorak and Chen [2-4] demonstrated that the MoriTanaka and self-consistent approximations may lead to two distinct sets of effective properties, and violate some rigorous bounds. At the present time, the computation of thermo-elastic properties for materials exhibiting this kind of microstructure still seems to constitute an open question.

\section{Computing the effective properties of the composite}

Effective properties of the reinforcing strips. The homogenization of the reinforcing strip corresponds to the case, treated in a recent paper [18], of an unidirectional fiber-reinforced composite ply. As a unique morphology is considered for every constituent of the reinforcing strip,

\begin{tabular}{|c|c|c|c|c|c|c|c|c|}
\hline & \multicolumn{6}{|c|}{ Mechanical moduli } & \multicolumn{2}{|c|}{ CTE } \\
\hline & $\begin{array}{c}\mathrm{E}_{\mathrm{x}} \\
{[\mathrm{GPa}]}\end{array}$ & $\begin{array}{l}\mathrm{E}_{\mathrm{y}}, \mathrm{E}_{\mathrm{z}} \\
{[\mathrm{GPa}]}\end{array}$ & $v_{x y}, v_{x z}$ & $\begin{array}{c}\mathrm{G}_{\mathrm{xy}}, \mathrm{G}_{\mathrm{xz}} \\
{[\mathrm{GPa}]}\end{array}$ & $v_{\mathrm{yz}}$ & $\begin{array}{c}\mathrm{G}_{\mathrm{yz}} \\
{[\mathrm{GPa}]}\end{array}$ & $\begin{array}{c}\alpha_{\mathrm{x}} \\
{\left[10^{-6} / \mathrm{K}\right]}\end{array}$ & $\begin{array}{c}\alpha_{\mathrm{y}}, \alpha_{\mathrm{z}} \\
{\left[10^{-6} / \mathrm{K}\right]}\end{array}$ \\
\hline Reinforcing strip & 146.8 & 10.2 & 0.274 & 7.0 & 0.355 & 3.8 & -0.620 & 48.0 \\
\hline T300 fibers [18] & 230 & 15 & 0.20 & 15 & 0.07 & 7 & -1.5 & 27 \\
\hline N5208 matrix [19] & 4.5 & 4.5 & 0.4 & 1.61 & 0.4 & 1.61 & 60 & 60 \\
\hline
\end{tabular}
the two above formulations of the self-consistent model lead to the same results. Consequently, one will only give the effective properties of the reinforcing strip (Table 1), accounting for a fiber volume ratio of $63 \%$.

Table 1: Thermomechanical properties of a reinforcing strip and its constituents.

Effective properties of the composite. The second homogenization step is more problematic, as it involves inclusions of ellipsoidal shapes presenting a random geometrical orientation, combined with a strong anisotropy of the constituents and the effective medium. As a consequence, the two formulations detailed above (Eq. 10-13) will be used to perform this second homogenization step. 
(Eq. 10) and (Eq. 12) provide the effective stiffness of the composite. The averaging operations are achieved onto the two previously described constituents (reinforcing strips with $95 \%$ volume ratio and extra-reinforcement matrix with $5 \%$ ratio). A set of 10 orientations, uniformly distributed on $180^{\circ}$ in the 1-2 plane (ply plane, see Fig. 1), is considered in order to account for the random orientation. The effective elastic moduli obtained are summed up in the Table 2 below. The notation Hmixed denotes the direction-dependent formulation, described below.

\begin{tabular}{|c|cccccc|}
\hline & $\begin{array}{c}\mathrm{E}_{1}, \mathrm{E}_{2} \\
{[\mathrm{GPa}]}\end{array}$ & $\begin{array}{c}\mathrm{E}_{3} \\
{[\mathrm{GPa}]}\end{array}$ & $\mathrm{v}_{12}$ & $\begin{array}{c}\mathrm{G}_{12} \\
{[\mathrm{GPa}]}\end{array}$ & $v_{13}, v_{23}$ & $\begin{array}{c}\mathrm{G}_{13}, \mathrm{G}_{23} \\
{[\mathrm{GPa}]}\end{array}$ \\
\hline \hline $\mathrm{H} \sigma$ & 55.48 & 11.24 & 0.290 & 21.49 & 0.266 & 3.97 \\
$\mathrm{H} \varepsilon$ & 16.63 & 9.92 & 0.121 & 7.42 & 0.337 & 4.45 \\
$\mathrm{Hmixed}$ & 55.41 & 11.20 & 0.289 & 21.49 & 0.279 & 4.496 \\
\hline Voigt bound & 55.46 & 11.28 & $\mathrm{X}$ & 21.49 & $\mathrm{X}$ & 5.20 \\
Reuss bound & 15.44 & 9.66 & $\mathrm{X}$ & 6.65 & $\mathrm{X}$ & 4.46 \\
\hline
\end{tabular}

Table 2: Estimated elastic moduli of the effective composite.

One may first notice that the two methods lead to drastically different stiffnesses, particularly for the components that govern the in-plane behaviour $\left(E_{1}, v_{12}\right.$ and $\left.G_{12}\right)$. On the contrary, the "out-ofplane" components $\left(\mathrm{E}_{3}, \mathrm{v}_{13}\right.$ and $\left.\mathrm{G}_{13}\right)$ do not vary very much from a homogenization procedure to the other. Moreover, these moduli respect, within the prescribed $10^{-3}$ accuracy, the Reuss and Voigt bounds (see Table 2). The elastic moduli obtained with the strain-based formulation are close to the Reuss bound; whereas those obtained with the stress-based formulation are practically merged with the Voigt bound. Yet, previous works achieved on composite laminates (which have a similar structure) suggested that extreme direction-dependent homogenization procedures gave satisfying results on both in-plane and out-of-plane behaviour.

A mixed homogenization scheme, inspired from the Vook-Witt model $[20,21]$ is thereby proposed. The in-plane behaviour is modeled using the stress-based formulation, whereas the strainbased formulation is used for the out-of-plane behaviour. With this mixed formulation, the stiffness tensor of the composite satisfies the form given in (Eq. 14) below. The results given by this formulation are also summed up in Table 2 above.

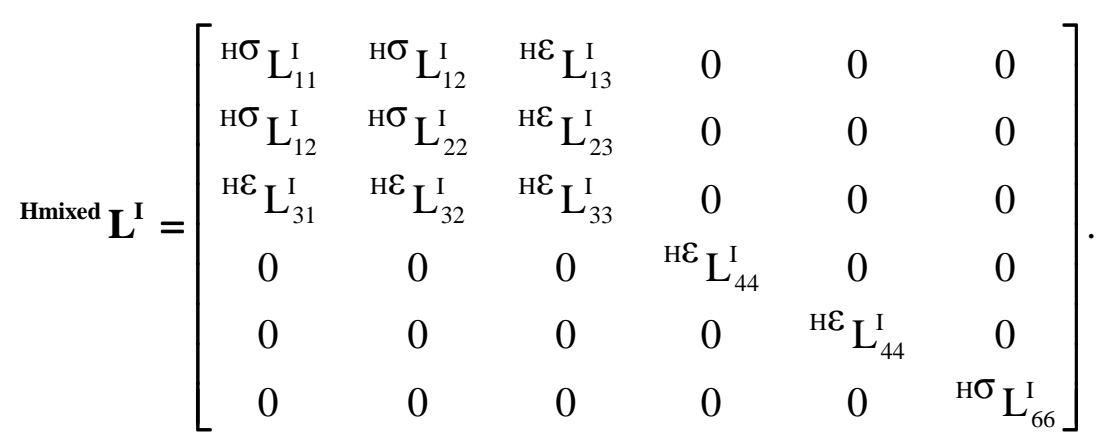

The computation of the averages for localization and concentration tensors, upon the two constituents and all the orientations, is a good indicator of the relevance of each formulation (see Table 3 below). For the two "pure" formulations (Ho and $\mathrm{H} \varepsilon)$, the in-plane components $(11,12$ and 66 components in Eq. 14) exhibit very important errors regarding Hill's averages principles; for them, the stress-based formulation $(\mathrm{H} \sigma)$ gives the lowest errors. On the contrary, for the out-of-plane components, the strain-based formulation $(\mathrm{H} \varepsilon)$ is the most reliable. The direction-dependent formulation combines the advantages of those two and guarantees a relative error lower than $3 \%$ on every term. 
It is important to notice that these errors are null for a penny-shaped morphology, whichever formulation is used. However, they quickly grow up with an increased stretching or thickness of the reinforcing strips, so the model is satisfying only for a limited range of morphologies.

\begin{tabular}{|c|ccccccc|}
\hline$\left\langle\mathbf{A}^{\mathbf{i}}\right\rangle$ & $\mathrm{A}_{11}=\mathrm{A}_{22}$ & $\mathrm{~A}_{33}$ & $\mathrm{~A}_{44}=\mathrm{A}_{55}$ & $\mathrm{~A}_{66}$ & $\mathrm{~A}_{12}=\mathrm{A}_{21}$ & $\mathrm{~A}_{13}=\mathrm{A}_{23}$ & $\mathrm{~A}_{31}=\mathrm{A}_{32}$ \\
$\mathrm{H} \varepsilon$ & 1.001 & 1 & 0.5 & 0.5 & 0 & 0 & 0 \\
$\mathrm{H} \sigma$ & 1.031 & 1 & 0.443 & 0.512 & 0.007 & 0 & -0.033 \\
$\mathrm{Hmixed}$ & 1.029 & 1 & 0.500 & 0.511 & 0.007 & 0 & -0.015 \\
\hline \hline$\left\langle\mathbf{B}^{\mathrm{i}}\right\rangle$ & $\mathrm{B}_{11}=\mathrm{B}_{22}$ & $\mathrm{~B}_{33}$ & $\mathrm{~B}_{44}=\mathrm{B}_{55}$ & $\mathrm{~B}_{66}$ & $\mathrm{~B}_{12}=\mathrm{B}_{21}$ & $\mathrm{~B}_{13}=\mathrm{B}_{23}$ & $\mathrm{~B}_{31}=\mathrm{B}_{32}$ \\
$\mathrm{H} \varepsilon$ & 3.465 & 1 & 0.5 & 1.431 & 0.604 & -1.178 & 0 \\
$\mathrm{H} \sigma$ & 1.000 & 1 & 0.5 & 0.5 & 0 & 0 & 0 \\
Hmixed & 1.001 & 1.001 & 0.497 & 0.5 & 0.001 & -0.009 & 0.001 \\
\hline \hline Expected & 1 & 1 & 0.5 & 0.5 & 0 & 0 & 0 \\
\hline
\end{tabular}

Table 3: Averages of the localization and concentration tensors, for the composite.

As above, the Coefficients of Thermal Expansion have been computed with respect to the three homogenization approaches previously presented (see Eq. 11 and Eq. 13). With the mixed formulation, the effective CTE satisfies the structure given below (Eq. 15). The stiffness obtained with each formulation was used for computing the corresponding CTE. This leads to the results presented in Table 4 below. As for the stiffness, a significant deviation between the results occurs, depending on the homogenization procedure used. This discrepancy remains if the same stiffness is used for the three formulations. In order to quantify the relevance of these results, the dimensionless errors $\mathbf{X}$ and $\mathbf{Y}$ were also defined as in (Eq. 16), and detailed in Table 4.

$$
\begin{aligned}
& \text { Hmixed } \boldsymbol{\alpha}^{\mathrm{I}}=\left[\begin{array}{ccc}
{ }^{\mathrm{\sigma}} \boldsymbol{\alpha}_{11}^{\mathrm{I}} & 0 & 0 \\
0 & { }^{\mathrm{N} \sigma} \alpha_{11}^{\mathrm{I}} & 0 \\
0 & 0 & { }^{\mathrm{E} \varepsilon} \alpha_{33}^{\mathrm{I}}
\end{array}\right] \\
& \left\{\begin{array}{l}
\mathbf{X}=\left\langle\mathbf{a}^{\mathrm{i}}\right\rangle \cdot\left(\boldsymbol{\alpha}^{\mathrm{I}} \Delta \mathrm{T}\right)^{-1} \\
\mathbf{Y}=\left\langle\mathbf{b}^{\mathrm{i}}\right\rangle \cdot\left(\mathbf{L}^{\mathrm{I}}: \boldsymbol{\alpha}^{\mathrm{I}} \Delta \mathrm{T}\right)^{-1}
\end{array}\right.
\end{aligned}
$$

One can observe that the strain-based formulation satisfies Hill's averages principle over the strains, but leads to some errors on thermal stresses and mainly in the normal direction. Respectively, the stress-based formulation satisfies Hill's averages principle over stresses but underestimates the in-plane thermal strains by more than $200 \%$. As observed for the elastic behaviour, the mixed formulation gives the best compromise between these two aspects, and leads to absolute errors lower than $3 \%$ on every term (increasing with the shape ratios of the reinforcing strips). This error vanishes if one prescribes a penny-shaped morphology to the constituents. This demonstrates that the classical self-consistent model is valid if and only if a single morphology is used for all the constituents in the Representative Elementary Volume. This fact is very important as, for this particular case, the property values obtained using a penny-shaped morphology and a stretched one are very close (with Hmixed formulation only), which opens a way for a significant simplification of the model. 


\begin{tabular}{|c|cc|cc|cc|}
\cline { 2 - 7 } & \multicolumn{2}{|c|}{ CTE } & \multicolumn{2}{c|}{ Error on $\varepsilon$} & \multicolumn{2}{c|}{ Error on $\sigma$} \\
& $\alpha_{1}, \alpha_{2}$ & $\alpha_{3}$ & $\mathrm{X}_{1}$ & $\mathrm{X}_{3}$ & $\mathrm{Y}_{1}$ & $\mathrm{Y}_{3}$ \\
& {$\left[10^{-6} / \mathrm{K}\right]$} & {$\left[10^{-6} / \mathrm{K}\right]$} & & & & \\
\hline $\mathrm{H} \varepsilon$ & 24.8 & 49.9 & 0 & 0 & -2.088 & 0 \\
$\mathrm{H} \sigma$ & 3.5 & 114.9 & 0.062 & -0.424 & 0 & 0 \\
Hmixed & 3.5 & 64.3 & 0.031 & 0.027 & 0.004 & 0.002 \\
\hline Expected [5] & $3.5 \sim 5$ & $45 \sim 50$ & 0 & 0 & 0 & 0 \\
\hline
\end{tabular}

Table 4: Estimated CTEs of the composite and associated errors.

A few experimental results can corroborate the estimated effective properties. The in-plane elastic modulus was measured to be approximately $42 \mathrm{GPa}$ [5], and the CTEs were also measured at room temperature (see Table 4). These values are in the same order of magnitude to those estimated with the scale transition procedure. The difference is due to the use of slightly different constituents (AS4 fibers and bismaleimide resin) and fiber ratio (53\%), and to out-of-plane waviness of the reinforcing strips (which deteriorate their in-plane apparent properties). A more realistic estimation is given in [22], for which the predicted properties match the measured ones to within $\pm 5 \%$.

\section{Application to thermo-mechanical loads}

Response of the composite to purely mechanical load. In order to describe the multi-scale mechanical behaviour of the composite, a macroscopic uniaxial in-plane traction of $100 \mathrm{MPa}$ is considered. (Eq. 7) is used to compute the local stresses in the constituents, dropped in the local coordinate system Ri.
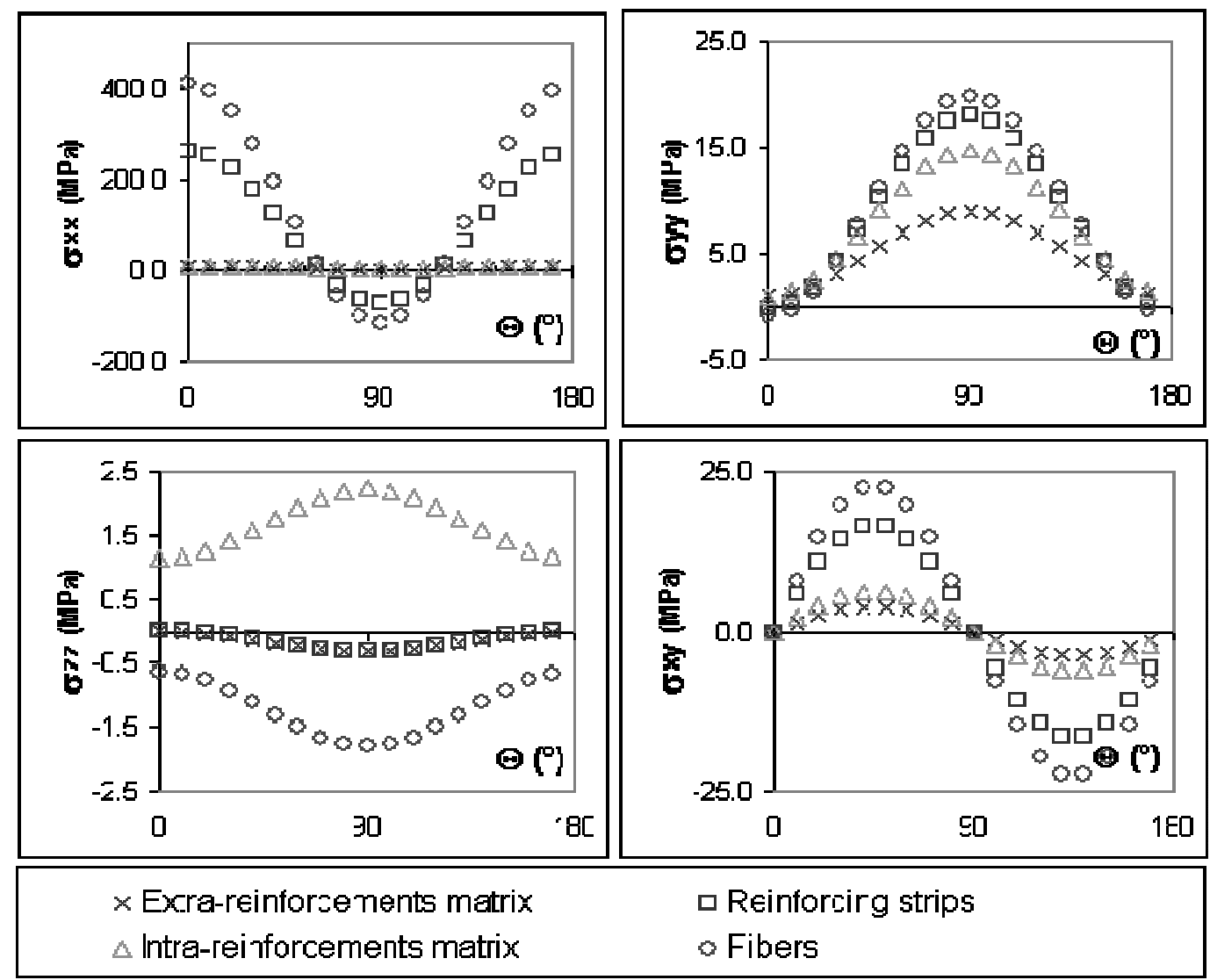

Figure 3: Local stress states in the composite under a $100 \mathrm{MPa}$ uniaxial traction.

One can observe (Fig. 3) that these stresses evolve with the orientation angle as $\pi$-periodic sinusoids. At the mesoscopic scale, the in-plane stresses are strongly heterogeneous (contrarily to 
the in-plane strains, which are rather homogeneous); the reinforcing strips experience up to 260 $\mathrm{MPa}$ in the x-direction, while the organic matrix undergoes less than $10 \mathrm{MPa}$. This concentration of stresses is a result of the orientation mismatch between the reinforcement strips and the loads. It is increased into the fiber constituting the reinforcement strips, which experience more than $400 \mathrm{MPa}$ while the matrix takes less than $20 \mathrm{MPa}$. On the other hand, out-of-plane stresses are very low: one will only notice the emergence of complementary stresses between the constituents of the reinforcing strips.

Response of the composite to purely thermal load. A similar study was achieved for the case of a macroscopically stress-free $-100{ }^{\circ} \mathrm{C}$ thermal load, which is typical of the cool-down that occurs after the curing stage of composite materials, and which induces severe residual stresses [23, 24]. Contrarily to the mechanical load studied previously, the thermal expansion respects the in-plane symmetry of the material, thus the shear stresses and strains are null, and the mechanical states are independent on the orientation angle $\Theta$ of the reinforcing strip. This result can be generalized to any load respecting the in-plane symmetry.

\begin{tabular}{|c|c|c|c|c|}
\hline \multirow{2}{*}{ Scale } & \multirow{2}{*}{ Medium } & \multicolumn{3}{|c|}{ Stresses $(\mathrm{MPa})$} \\
\hline & & $\sigma_{\mathrm{Xx}}$ & $\sigma_{\mathrm{yy}}$ & $\sigma_{\mathrm{zZ}}$ \\
\hline Macroscopic & Composite & 0.0 & 0.0 & 0.0 \\
\hline \multirow{2}{*}{ Mesoscopic } & Extra-reinforcement matrix & 42.6 & 42.5 & 0.4 \\
\hline & Reinforcing strips & -48.1 & 44.1 & 0.1 \\
\hline \multirow{2}{*}{ Microscopic } & Intra-reinforcement matrix & 57.79 & 55.51 & 25.39 \\
\hline & Fibers & -109.9 & 37.5 & -14.7 \\
\hline
\end{tabular}

Table 5: Stress states in the composite and its constituents exposed to a $-100{ }^{\circ} \mathrm{C}$ thermal load.

The local stresses inside the composite are summed up in the Table 5 above. At the mesoscopic scale, one can observe a marked gap between the $\sigma_{\mathrm{xx}}$ stresses in the two constituents: the matrix undergoes traction stresses, although the reinforcing strips are compressed (which implies a risk of meso-buckling). The same scheme appears at the microscopic scale: the fibers are compressed and the matrix stretched. Along the normal direction $\mathrm{z}$, one can also notice the emergence of nonnegligible complementary stresses in the fibers and the matrix, due to the gap of properties.

\section{Discussion and perspectives}

A two-steps scale transition procedure based on the self-consistent model has been introduced to describe the thermo-mechanical behaviour of a composite material reinforced by inclusions randomly oriented in the ply plane. The limits of the self-consistent model for this kind of microstructures have been discussed on the base of Hill's averages principles, which were used to define error estimators. Those results inspired the use of a mixed direction-dependent formulation, which enables to drastically reduce the error for this particular material and microstructure.

This method may also be applicable to other particles-reinforced materials such as short-fibers reinforcements, or nanocomposites (as an interesting alternative to the Krenchel model [25, 26]). More generally, for materials constituted by inclusions with very distinct shapes and/or alignment, a special attention must be paid to the uniqueness of estimated properties and the satisfaction of Hill's averages principles.

In a second time, this mixed formulation has been applied to the simulation of local stresses in the constituents of the material, when subjected to thermal of mechanical loadings. For uniaxial loading, a dependency of the local stresses on the orientation angle between the reinforcing strips and the direction of solicitation was found, along with high stress levels into the rigid elements. On the other hand, thermal loading (cooling) seems to tax mainly the matrix with tensile stresses, while the fibers are subjected to compressive stresses that might induce micro-buckling effects. However, 
as the self-consistent model only provides average values of the local stress fields, its application to the determination of material health is somewhat limited.

A direct application of this work is the simulation the development of residual stresses during the manufacturing process of carbon-epoxy composites [22]. The very low computational time cost of the self-consistent method may be an advantage for this kind of simulations, where the very nonlinear behavior of the resin during the fabrication process (due to hardening, thermo-chemical shrinkage, dependency with temperature...), implies repeated computations of the effective properties.

\section{References}

[1] U.F. Kocks, C.N. Tomé and H.R. Wenk: Texture and anisotropy (1998), Cambridge University Press.

[2] Y. Benveniste: A new approach to the application of Mori-Tanaka's theory in composite materials, Mechanics of Materials, Vol. 6 (1987), pp. 147-157.

[3] Y. Benveniste, G.J. Dvorak and T. Chen: On diagonal and elastic symmetry of the approximate effective stiffness tensor of heterogeneous media, Journal of Mechanics and Physics of Solids, Vol. 39 (1991), pp.927-946.

[4] T. Chen, G.J. Dvorak and Y. Benveniste: Mori-Tanaka estimates of the overall elastic moduli of certain composite materials, Journal of Applied Mechanics, Vol. 59 (1992), pp. 539-546.

[5] Hexcel France: Hextool Datasheet (information on www.hexcel.com).

[6] R. Hill: The essential structure of constitutive laws for metals composites and polycrystals, Journal of the Mechanics and Physics of Solids, Vol. 15 (1967), pp. 79-95.

[7] J.D. Eshelby: The Determination of the Elastic Field of an Ellipsoidal Inclusion, and Related Problems, Proceedings of the Royal Society London, Issue A241 (1957), pp. 376-396.

[8] R. Hill: The essential structure of constitutive laws for metals composites and polycrystals, Journal of the Mechanics and Physics of Solids, Vol. 13 (1965), pp. 89-101.

[9] R. Morris: Elastic constants of polycrystals, International Journal of Engineering Science, Vol. 8 (1970), pp. 49-61.

[10] S. Fréour, F. Jacquemin and R. Guillén: Extension of Mori-Tanaka approach to hygro-elastic loading of fiber-reinforced Composites - Comparison with Eshelby-Kröner self-consistent model, Journal of Reinforced Plastics and Composites, Vol. 25 (2006), pp. 1039-1052.

[11] J. Berryman, P. Berge: Critique of two explicit schemes for estimating elastic properties of multiphase composites, Mechanics of Materials, Vol. 22 (1996), pp. 149-164.

[12]E. Kröner: Berechnung der elastischen Konstanten des Vielkristalls aus des Konstanten des Einkristalls, Zeitschrift für Physik, Vol. 151 (1958), pp. 504-508.

[13]E. Kröner: Zur plastischen verformung des vielkristalls, Acta Metallurgica, Vol. 9 (1961), pp.155-161.

[14] J.W. Hutchinson: Elastic-plastic behaviour of polycrystalline metals and composites, Proceedings of the Royal Society London, issue 319 (1970), pp. 247-272.

[15] G.J. Weng: A self-consistent relation for the time-dependent creep of polycrystals, International Journal of Plasticity, Vol. 9 (1993), pp. 181-198. 
[16]E. Le Pen, D. Baptiste: Multi-scale fatigue behaviour modelling of Al/Al2O3 short fibre composites by a micro-macro approach, International Journal of Fatigue, Vol. 24 (2002), pp. 205-214.

[17]D. Baptiste: Non Linear Behavior Micromechanical Multi-scale Modelling of Discontinuous Reinforced Composites, Materials Science Forum, Vol. 426-432 (2003), pp. 3939-3944.

[18]F. Jacquemin, S. Fréour and R. Guillén: A hygro-elastic self-consistent model for fiberreinforced composites, Journal of Reinforced Plastics and Composites, Vol. 24 (2005), 485502.

[19] A. Agbossou, J. Pastor: Thermal stresses and thermal expansion coefficients of n-layered fiberreinforced composites, Composite Science and Technology, Vol. 57 (1997), pp.249-260.

[20]R.W. Vook, F. Witt: Thermally induced strains in cubic metal films, Journal of Applied Physics, Vol. 39 (1968), pp. 2773-2776.

[21]U. Welzel, S. Fréour: Extension of the Vook-Witt and inverse Vook-Witt elastic graininteraction models to general loading states, Philosophical Magazine, Vol. 87 (2007), pp. 3921-3943.

[22]E. Lacoste, K. Szymanska, S. Terekhina, S. Fréour, F. Jacquemin, M. Salvia: A multi-scale analysis of local stresses during the cure of a composite tooling material, In Press (Composites Part A).

[23] J.A. Guemes: Curing Residual Stresses and Failure Analysis in Composite Cylinders, Journal of Reinforced Plastics and Composites, 13 (1994), pp. 408-419.

[24] K. Ogi, H.S. Kim, T. Maruyama and Y. Takao: The infuence of hygrothermal conditions on the damage processes in quasi-isotropic carbon/epoxy laminates, Composites Science and Technology, Vol. 59 (1999), p. 2375.

[25]H. Krenchel, in: Fibre Reinforcements (1964), Akademisk Forlag, Copenhagen, Denmark.

[26]E.T. Thostenson, T.W. Chou: On the elastic properties of carbon nanotube-based composites: modeling and characterisation, Journal of Physics D: Applied Physics, Vol. 36 (2003), pp. 573582. 